\title{
An Automated Research for Emotion Recognition and Generation
}

\author{
Vivek Venugopal, M.R. Stalin John, Vasanth Kumar. CH
}

\begin{abstract}
As of today, Human Robot Interaction (HRI) is considered as an on-going and challenging trend. The development in this field is vast and still in budding phase with respect to research. Several Algorithms and Models are being designed that allow the robots to precept think and behave like a human being. Scientists are working regressively on creating an 'Artificial Mind' as an infrastructure using computational methods which are specifically delineated to guide a robot. Artificial Intelligence has taken the next level which is called as 'Embodied Artificial Intelligence' in order to make the humanoids smarter. This research deals with, a practical robot head which recognizes and mirrors the same basic emotions from the user with the presence of visual inputs and Facial Action Code System (FACS) control points on the human face. This research work deals with the design and development of three major modules in which, one module involves the fabrication of the practical robot head to represent the emotions. The next major module is the Artificial Intelligence segment including a Fuzzy Logic. In this segment, the inputs will be taken from the user in the form intensities of visual signals. Based on the Fuzzy Logic classifications; the respective emotion from the user is recognized and fed forward to the practical head. The third module of this research deals with the interfacing mechanism between the Fuzzy Logic module and the Mechanical Humanoid head for traversing the signals generated from the Fuzzy Logic Chunk to the practical robot head in the form mechanical actions. This proposed research model will have a huge impact in the study of interaction between the Human and Robots and research studies on certain critical diseases or syndromes like ASD, Alzheimer's disease, and Bell's palsy.
\end{abstract}

Keywords - Artificial Intelligence; Human Robot Interaction (HRI); Artificial Mind; Fuzzy Logic and Facial Action Code System (FACS).

\section{INTRODUCTION}

Recent studies have shown that, daily life activities are becoming prevalent with the involvement of social robots and its outspreading to the commercial environment at faster rates. To scale up the interrelationship between Human and Robots, a vital role is played by Human-Robot Interaction (HRI) which deals with the study of interactions that can happen between a Human Being(s) and a Robot(s). Cognitive robotics study, which is a crucial area in HRI, tends to implement the respective models into the robot and will allow us to study the robot action and finally the model with which the robot mimics the human action closely, can be selected, from available models for a solution[18,19].

Vivek Venugopal, Department of Mechanical Engineering SRM Institute of Science and Technology Chennai, Tamil Nadu, India. (E-mail: vivek_venugopal@srmuniv.edu.in)

M.R. Stalin John, Department of Mechanical Engineering SRM Institute of Science and Technology Chennai, Tamil Nadu, India. (E-mail: stalinjohn.m@ktr.srmuniv.ac.in)

Vasanth Kumar. CH, Department of Mechanical Engineering SRM Institute of Science and Technology Chennai, Tamil Nadu, India. (E-mail: vasanthkumar.s@ktr.srmuniv.ac.in)
Revised Manuscript Received on July 10, 2019.

There is a closely knit functional relationship between emotion and social cognition. Socio-Cognitive Robotics is a highly appreciated field of robotics as it is mainly based on the mimicking concepts. This paves the way for the systems to understand the various diseases or syndromes in patients at all age groups such as ASDs, Alzheimer's disease etc. for which there is no exact cure. "An Artificial Brain" which is the critical area of this research is a system comprising of the software and hardware which has cognitive abilities which is similar to those of a human being's brain or to that of the brain of an animal.

Prince and Suliman [1] proposed the Artificial Brain Emotion Recognition and Generation System (ABERGS) and described a method for recognizing the emotion from the human being and generating as a robotic mood state using Fuzzy Kohonen Clustering Network (FKCN) logic. Wagshum et al., [2] reported a new mechanism design for the humanoid robot head with the basic facial expressions. The approach is a cost effective one and with lesser complexities. Also, there is a reduction in the number of actuators used for generating the facial expressions compared to the previous studies done in this area of research. Bezdek et al., [3] developed Fuzzy Kohonen Clustering Networks (FKCN) and explained about the algorithm. The following algorithm was developed to resolve several major issues faced by the Kohonen Clustering Network. Nicole et al., [4] explained the different aspects of designing a mind of a social robot. The author provided an introduction for the researchers who are in the process of designing the mind and brain of the social or an emotional robot. As part of his research, a humanoid called as Facial Automation for Conveying Emotions (FACE) robot was created which has the demonstrated the results expected from the research perspective in a satisfactory manner. Jong et al., [5] designed an artificial face for the humanoid which acts a gateway for the interactions using emotions between the human and robot. The author provided the mechanism for the generation of the facial expression which was an imitation of the same expression or the mood state that was shown by the human being who was the part of the activity. Ali et al., [6] described and designed a method, about the role of embodiment and presence in human perception of robots. It brought an approach in understanding how the human beings respond to the virtual and physical agents, which was considered to be the different forms of social robots. Terrence et al., [7] surveyed and reviewed the available socially interactive robots as part

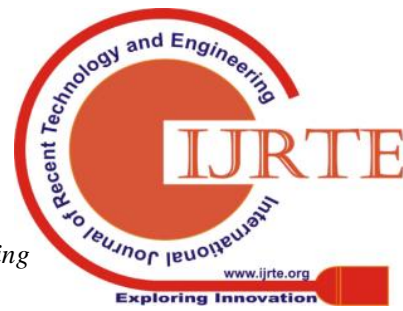




\section{AN AUTOMATED RESEARCH FOR EMOTION RECOGNITION AND GENERATION}

of the research and concluded that the socially interactive robots will play a crucial role in our world while working with humans and while working for the human beings. The author highlighted the involvement of the socially interactive robots in the field of health care, therapy and rehabilitation.

Yu and Xiaojiao, [8], studied and analyzedthe simulations of humanoid facial expression behavior and succeeded in designing the structure of the expression robot using Finite Element Analysis (FEA) software. The FEA software used for this purpose was ABAQUS which paved the way to analyze the four kinds of basic and typical facial expressions on the robot. Jizheng et al., [9] described about the designing of the head of the humanoid robot based on Uncanny Valley and FACS. In this paper, based on the survey done on the interaction of the robot head with the human beings, they could conclude that a robot head designed with 15 servo motors could show only the basic sets of human emotions. Ying-Li et al., [10] conducted an elaborated study on the facial expression analysis and recognizing the action units for the respective analysis. This study imparted a greater knowledge in identifying the different types of emotions generated by the human face and the same can be implemented in the social robot as well based on the Action Units (AU) of the face, which closely. Ge et al., [11] proposed a system which was highly interactive and be responsible for the reconstruction of the head. The research employed a non-linear mass spring movements during the facial expressions by coupling the (SVM) classifier to generate facial expressions which was more effective than that generated by the non-linear spring model. Meng-Ju et al., [12] developed a novel design of an autonomous robotic facial expression generation using Fuzzy Kohonen Clustering Network (FKCN) as its artificial brain. Lei et al., [13], presented a novel Adaptive Fuzzy Kohonen Clustering Network with algorithm for image segmentation purpose, to resolve the setbacks faced by the Kohonen Clustering Network alone for better convergence rates, reduced computational costs after comparing it with the conventional FKCN algorithm. Yanqing et al., [14], developed and demonstrated an algorithm for the Fuzzy Kohonen Clustering Network which has better efficiency than the conventional FKCN. The developed algorithm overcame the conventional FKCN making it three times faster than the original FKCN and could handle larger data sets which could not be handled by the original FKCN. Puri et al., [15] described the methodology used to detect facial expressions using image processing in Python. The research described the various steps from setting up the Python libraries required in this regard till the final output where the facial expression was detected.Viola and Jones, [16], described the technique of rapid object detection using boosted cascading method of simple features which can be used for the detection of the face as an input to the system. This technique helps in designing the algorithm for the software component of the research work. resembles with the expression shown by the humans pretty facial expression of a human being with the help of robot model for the simulation of around 22 facial muscles facial linear spring model with Support Vector Machine

The literature survey resulted in understanding the various autonomous methods for mimicking the facial expression of a human on a robot face with various simulation techniques. Also the methodology for the use of Fuzzy Logic for computing the input variables as part of the respective research and produce the output in the form of various simulation techniques have been discussed in the respective research papers. The research work done in papers on the autonomous facial expression recognitions lacked a particular criterion where the simulation techniques did not include the application of the logic on practical Robot faces but on visual types of simulation techniques. Hence, the research works lacked in the use of any microcontroller for the interfacing purposes. As part of this research work,the authors are concentrating on interfacing a practical robot head for mirroring the human emotion to an extent with the use of an efficient microcontroller. The logic considered for this research purpose will be Fuzzy Logic using Keras model with Haar Cascade Algorithm [16] which has got a better throughput of the system. Even the error rates of the dataset clusters are reduced significantly.The author concentrated on the fabrication of the practical robot head structure. The parts of the following robot head structure was completely built in-house and the fabrication was successfully completed with very minimal costs compared to that of the existing mechanisms as per the current trends

\section{METHODOLOGY}

The methodology that has been followed for this research has been diagrammatically shown in Fig. 1, the methodology provides and overall functionality of the respective research activity.

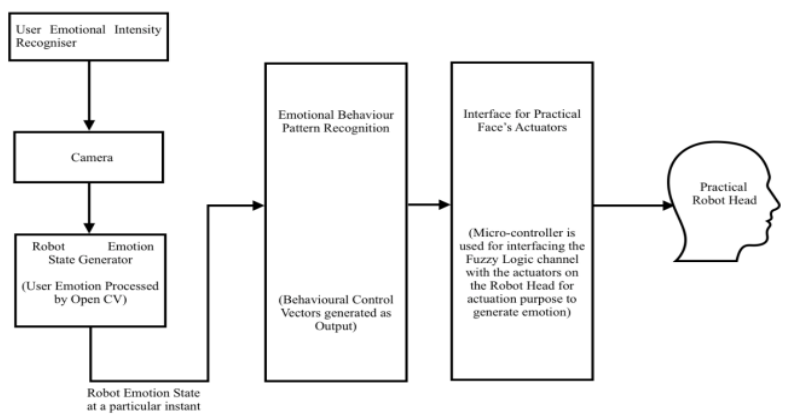

Fig. 1: Block Diagram for Methodology

\section{A. User Emotional Intensity Recognizer:}

The emotion state of the face of the human being is captured. The image sensing device or the camera is placed in front of the human face while the image is captured. The Open CV software will be able to identify the intensities of the emotion of the human face at a particular time $(t)$ is identified and is represented as vectors and is passed to the next stage of setup. The range of the intensities fed as an input ranges between 0 and 1 . The intensity values are represented as specific values which vary for each emotions represented by the human being which can be Happiness, Fear, Sadness or Neutral etc. 


\section{B. Robot Emotion State Generator:}

The values of the facial expression intensity, at a particular time are fed and have got the logic to generate the Emotion State at that particular moment of time. The output generated from this particular block is a behavioral vector which is fed to the next block or layer of the setup of the project. Here the Robotic Mood State at a particular instant which is the output of the respective is updated and represented as $\left(\mathrm{E}_{\mathrm{i}}\right)$ and the mood state of the robot at a previous instant is stored in the buffer.

\section{Emotional Behavior Pattern Recognizer:}

The most critical block of the setup or the methodology and is considered as the artificial brain setup of the robot which leads to the pattern recognition. Fusion Designing is carried out in this block is by using the concept of Pattern Recognition. Fuzzy Logic is used for analyzing the type of the emotion shown by the user to the camera setup. Once the camera captures the image, the same is processed by the use of the Fuzzy Logic implemented to the setup by using Python with Tensor Flow. The Keras Model with Haar Cascade Algorithm was used to generate the output behavioral vectors. This output from the module will be certain set of values which is considered as vectors. It is also called as the behavioral vectors in specific which is fed to the interfacing unit between the practical robot head and front end applications.

\section{Interface for Practical Face Actuators:}

The signal generated from the previous block of the setup of the robot 'Emotional Behavior Pattern Recognition' will have to converted to a pulse signal for the motors prepared for the actuation purpose of the respective emotions on the practical face of the robot head structure. The microcontroller facility selected for this purpose can be an ARDUINO MEGA 2560 which enables the communication with the practical robot head's artificial face actuators. Each motor assigned for the respective emotion that is to be mirrored by the physical robot face simulator is connected to the respective ports of the microcontroller whichever is going to be used and the microcontroller should be given the necessary power supply as per requirements.

\section{1) Practical Robot Head with Face Simulator:}

For this work and methodology the author decided on building a practical robotic face for mirroring the emotions as made by the user or the human being. The input from the controller interface between the fuzzy logic and the physical robotic head is fed to the respective motor whose blades are connected to the respective action points on the face of the robot via tendons and springs. On the actuation of the motor, which is actually a servo motor, the blade will rotate to the programmed angular degrees. On this motion the tendons will be pulled and the respective emotion will be displayed in on the robotic face on which the skin is applied for expressing the emotions after the actuation of the motors. The skin material is SILOCZEST LSR-105 RTV Silicon Rubber material. The skull for the face was made with the PLA (Poly Lactic Acid) material via 3D Printing. For each emotion to be displayed on the face of the robot, each motor was set on a pulley setup for the pulley action on the tendons connected between the motor and the face's action points. Also to prepare the skin of the robot's practical face, a mold was prepared which had a curing period of a week and the skin material was made through the mold which was prepared. Fig. 2 represents the mechanism followed by the robot head structure used for this research.

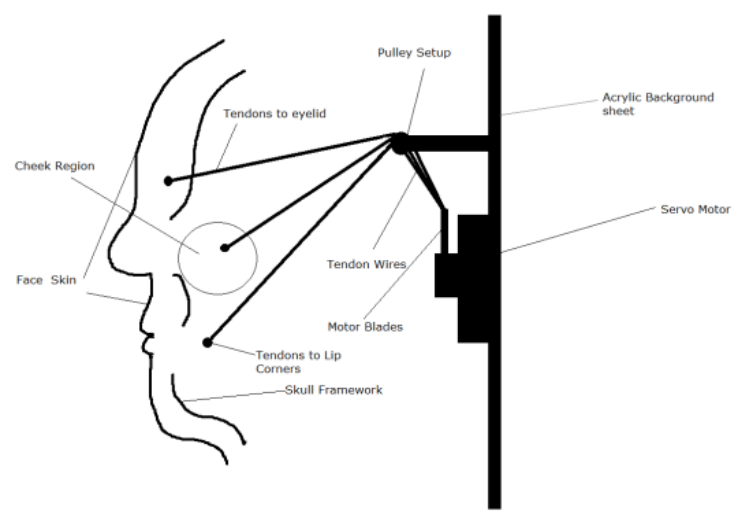

Fig. 2: Practical Robot Head Mechanism

\section{EXPERIMENTAL SETUP ARRANGEMENTS}

This research work had been done based on the fabrication perspective and software perspective. Apart from aforementioned activities in the experimental setup perspective, several other activities had to be carried out. These activities included the mold preparation for the creation of the mask of the practical Robot Head structure, creation of the face structure's skin material, identifying the Facial Action points etc. The detailed structural chart of the activities carried out as per process for this research has been shown in Fig. 3:

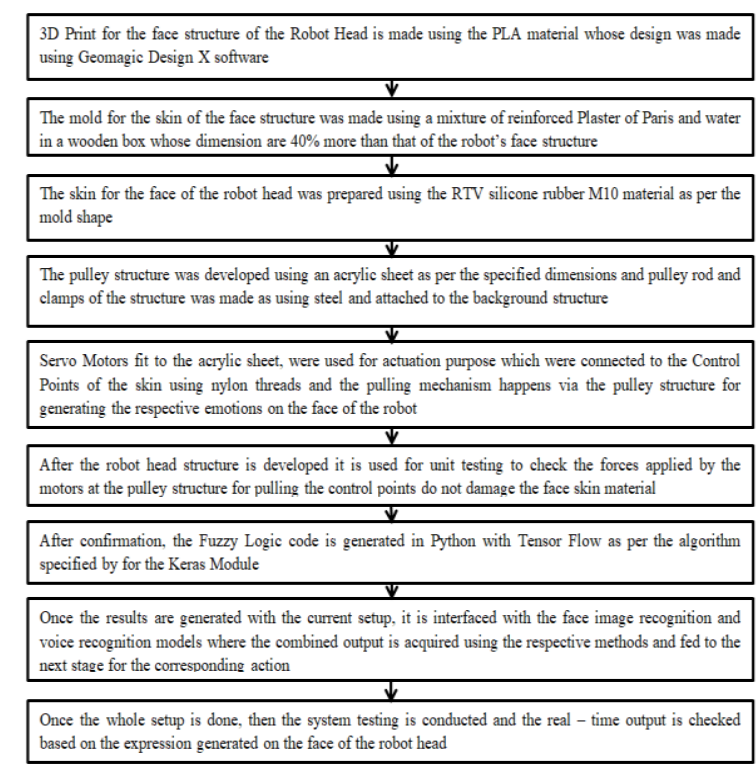

Fig. 3: Experimental Setup Steps

\section{A. Robot Head Development:}

The Robot Head had to be developed in in such a way that it came close to a realistic resemblance of a humanoid. 


\section{AN AUTOMATED RESEARCH FOR EMOTION RECOGNITION AND GENERATION}

\section{B. Face Structure:}

In this research, to develop the robot head, initially the author created the face skull structure using the STL file of an original skull of a human body. Modifications were made to the originally acquired file in GEOMAGIC DESIGN $X$ Software, based on the requirements from the research perspective. Once the modified STL file was generated, the final robot head face structure was generated using a Delta 3D printer and the material used for this activity was PLA.

As part of the preparation of the mold for the skin of the face mask of the project, a wooden box was made as the initial process. The dimensions of the wooden box was made keeping an extra $40 \%$ of the dimension of the face skull length wise, breadth wise and height wise. Initially half of the box was filled with sand and made wet for leveling purpose along with traces of hay over the sand bed. The face mask or skull on which oil was applied was fixed steadily on the leveled sand. Later a mixture of Plaster of Paris and water in the ratio (1:2) was applied to the face mask and spread over accordingly as per the shape. Also strays of coir was applied in the Plaster of Paris mixture for proper curing purpose of the mold.

\section{Background Pulley Mechanism:}

In this research, an acrylic sheet with the respective markings for the pulley set up and the motor fittings was cut and made ready. A clamp of $15 \mathrm{~cm}$ length was fit at each ends which is $30 \mathrm{~cm}$ apart from each other. A pipe rod of $1 \mathrm{~cm}$ diameter was welded to the end of each clamps fit to the acrylic sheet which is $30 \mathrm{~cm}$ apart. The motors (servo motors) for each expression of the face will be fit to the acrylic sheet at the positions as marked on it. The tendons which are actually the nylon wires will be tied from the motor blades and via the pulley mechanism will be joined to the action points of the face. Based on the rotation actionof the motor the wires will be pulled and released via the pulley mechanism.

\section{Fuzzy Logic in Python with Tensor Flow Application:}

In this research, the most critical zone was that of the use of the Fuzzy Logic method [15]. Python with Tensor Flow techniquefor coding was used to test the image processing of the three different emotions of three different users. The model used for this application is Keras Module with Haar Cascade Algorithm [16]. This helps in calculating the accuracy of out methodology to be used for the initial level of the image processing before feeding it to the next level of the setup for the robot face to mirror or imitate. The authoris trying to improvise our approach by implementing various combinations of "Emotional Seed" like \{happy, angry, compassionate\}; \{caring, sad, funny\}; \{smiling, rude, boring\}; \{mischievous, serious, funny\}; \{happy, naughty, angry\} etc. The fuzzy weights can vary between 0 and 1 theoretically; but in contrast to the research work done currently the author can also take weights in third place of decimal, e.g.: $0.548,0.876$ etc.

The intensities of the human emotions are collected, namely, neutral, happiness, sad and anger intensities are collected via Image sensing component [12]. The user's emotional state is identified by the combination of the emotional intensities values and is sent to the Emotional
Behavior Pattern Recognizer. This scheme has been designed using a fuzzy logic using Keras Model along with Haar Cascade Algorithm [16] for the autonomous generation of the facial expression on the robot face.The Python along with Tensor Flow running in the background was used as the platform for the coding purpose of the algorithm. The intensities of the image of the user are measured by the Fuzzy logic. The response is generated by the Fuzzy Logic in response to the estimated emotional intensities and is sent to the next stage. Finally the algorithm [16] for the Fuzzy Logic will be applied on the values obtained to give the final output after pattern recognition stage and is fed to the interface to simulate on the practical robot face.

\section{ALGORITHM}

The algorithm was designed based on the Haar Cascade Classifier and the use of Keras Model with Haar Cascade Algorithm for Object Detection Purpose (Face Recognition) [16]. The respective algorithm that was used for the face detection along with the detection of the emotion type of the face as part of this research activity is as follows:

Step 1: Load the required XML classifiers

Step 2: Load the input image (or video) - the human face in this case, in a grey scale mode

Step 3: Check if the face is found

Step 4: If yes, return the positions of the detected face as $\operatorname{Rect}(\mathrm{x}, \mathrm{y}, \mathrm{w}, \mathrm{h})$

Step 5: Once these locations are identified, the 'Region of Interest' (ROI) can be created for the face

Step 6: Based on the values of the pixels of the image created, the predictions are made - Prediction probability vector

Step 7: Find the maximum indexed array based on the predictions

Step 8: Define the list of emotions that are to be recognized and generated on the practical robot head

Step 9: Get the emotion value from the maximum indexed values of the variable assigned to the list of emotions - Most probable item

Step 10: Sent the respective value of the variable for emotions to the ARDUINO IDE through serial communication

Step 11: Get the output to the assigned motor and generate the respective type of emotion on the practical robot face

\section{EXPERIMENTAL SETUP}

The experimental setup was developed as three different modules, (i) Fabrication of the practical robot head module, (ii) Interfacing module between the front end input collection module and mechanical robot head module and finally (iii) The front end input module where the inputs was taken from the emotion of the human in the form of face image recognition intensities. The Robot head fabrication was considered as a novelty of this research work and the whole experimental setup can be represented as in Fig.4:

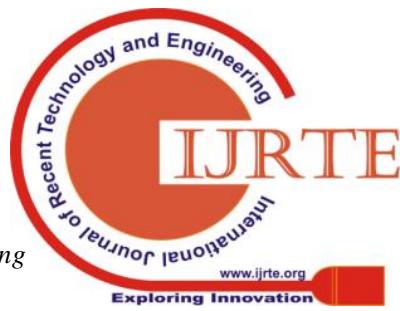




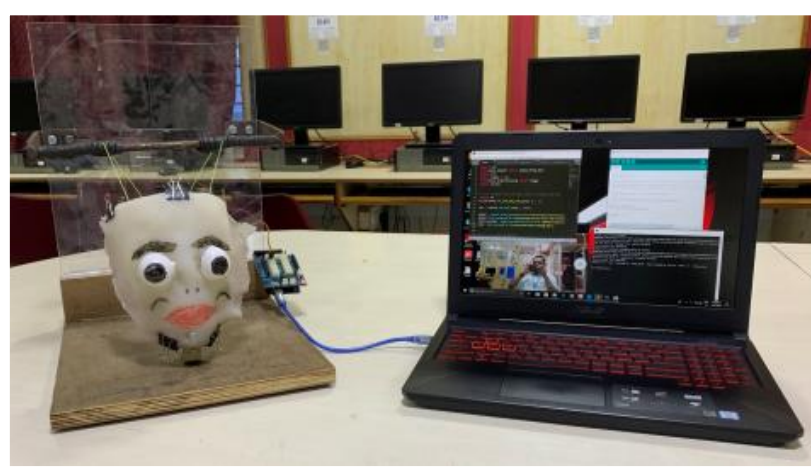

Fig. 4: Experimental Setup

\section{EXPERIMENTAL RESULTS}

The experiment was conducted as per process, for identifying the accuracy of the Fuzzy Logic method used for identifying the emotion types of different users (three in this case) and the author tried out 4 different emotions (Happy, Sad, Angry and Neutral). Based on the outcome of the fuzzy logic applied the author could calculate the accuracy of the fuzzy logic applied to the different emotions sets shown by different users Table 1 shows the Fuzzy Logic output for the emotions generated by different users.

Table 1: Fuzzy Logic output for the emotions generated by different subjects

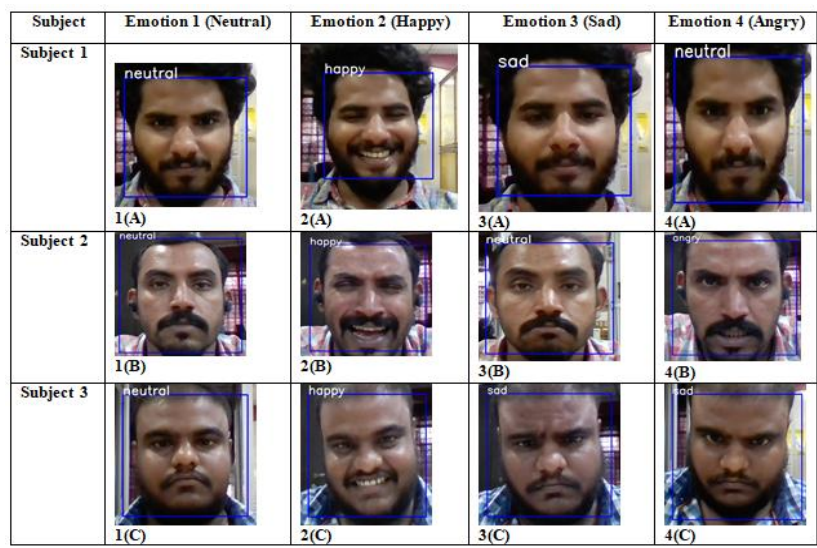

VII. CONCLUSION

In the current scenario of the research work, the author could fabricate a practical robot head with in-house developed Face Structure (using 3D Printing), Pulley Mechanism, Face Mask (Made using the mold and the material being SILOCZEST LSR-105 Silicone Rubber) and a Futuba S3003 Servo Motor with a sweep angle of $180^{\circ}$. And, the author could achieve this feat with very minimal costs incurred and a successful design phase test of the Practical Robot Head. The authors could also generate the intensities for the emotions generated by different users which could act as the input to the interface consisting of the actuators to generate the emotions on the face of the robot head. The author could achieve $75 \%$ of accuracy in each subject's emotion analysis by the fuzzy logic.

\section{ACKNOWLEDGMENT}

The authors of this research work sincerely thank Mr. D. Raja (Assistant Professor, Department of Mechanical
Engineering, SRMIST) and Mr. J. Daniel Glad Stephen (Assistant Professor, Department of Mechanical Engineering, SRMIST) for providing us support in preparing and developing the STL file for the skull framework for the robot head. The authors also express sincere gratitude towards the FABLAB team of SRMIST who extended their valuable support in generating the $3 \mathrm{D}$ printed model of the Robot Head Framework. The authors also thank Mr. E. Vijayaragavan (Assistant Professor, Department of Mechanical Engineering, SRMIST) for assisting us in the designing of the Backend Pulley Mechanism in CAD.

\section{REFERENCES}

1. M. Prince and S. Alsuhaibani, Adaptive artificial brain for humanoid robot using Pattern Recognition and Machine Learning, IJCSNS International Journal of Computer Science and Network Security, VOL.17 No.5, 2017.

2. W.T. Asheber, C.Y. Lin and S.H. Yen, Humanoid Head Face Mechanism with Expandable Facial Expressions, International Journal of Advanced Robotic Systems, DOI: 10.5772/62181, 2015.

3. J.C. Bezdec, C.K.E. Tsao and N.R. Pal, Fuzzy Kohonen Clustering Networks, [1992 Proceedings] IEEE International Conference on Fuzzy Systems, DOI 10.1109/FUZZY.1992.258797, 1992.

4. N. Lazzeri, D. Mazzei, L. Cominelli, A. Cisternino and D.E De Rossi, Designing the Mind of a Social Robot, MDPI/Journal/Applied Sciences, Vol. 302, DOI: 10.3390/app8020302, 2018.

5. J.W. Kwak, H.J. Chi, K.M. Jung, J.C. Koo, J.W. Jeon, Y. Lee et al., A Face Robot Actuated With Artificial Muscle Based on Dielectric Elastomer, Journal of Mechanical Science and Technology, Vol.19, No 2, pp. 578 588, 2005.

6. A. Mollahosseini, H. Abdollahi, T.D. Sweeny, R. Cole and M.H. Mahoor, Role of embodiment and presence in human perception of robots' facial cues, International Journal Human Computer Studies, Vol.116, pp. 25 39, 2018.

7. T. Fong, I. Nourbakhsh, and K. Dautenhahn, A survey of socially interactive robots, Robotics and Autonomous Systems, Vol. 42, pp. 143 166, 2003.

8. Y. Zhang and X. Wang, Analysis and Research of Humanoid Robot Facial Expression Behavior Simulations, Advanced Materials Research, ISSN: 1662-8985, Vol. 645, pp. 239 242, 2013.

9. J. Yan, Z. Wang and Y. Yan, Humanoid Robot Head Design Based on Uncanny Valley and FACS, Journal of Robotics, Vol. 2014, 5 pages, DOI: 10.1155/2014/208924, 2014.

10. Y.L. Tian, T. Kanade and J.F. Cohn, Recognizing Action Units for Facial Expressions Analysis, IEEE Transactions on Pattern Analysis and Machine Intelligence, Vol. 23, No 2, 2001.

11. S.S. Ge, C. Wang and C.C. Hang, Facial expression imitation in robot interaction, RO-MAN 2008 - The 17th IEEE International Symposium on Robot and Human Interactive Communication, DOI: 10.1109/ROMAN.2008.4600668, 2008.

12. M.J. Han, C.H. Lin and K.T. Song, Autonomous Emotional Expression Generation of a Robotic Face, Proceedings of the 2009 IEEE International Conference on Systems, Man, and Cybernetics, 978-1-4244-2794-9/09 IEEE, 2009.

13. W. Lei and Q. Feihu, Adaptive Fuzzy Kohonen Clustering Network for Image Segmentation, DOI: 0-7803-5529-6/99/ 1999 IEEE, 1999.
Blue Eyes Intelligence Engineering \& Sciences Publication 
14. Y. Yang, Z. Jia, C. Chang, X. Qin, T. Li, H. Wang et al., An Efficient Fuzzy Kohonen Clustering Network Algorithm, Fifth International Conference on Fuzzy Systems and Knowledge Discovery, 978-0-7695-3305-6/08 2008 IEEE, DOI 10.1109/FSKD.2008.91, 2008.

15. R. Puri, A. Gupta, M. Sikiri, M. Tiwari, N. Pathak, and S. Goel, Image Detection using Image Processing in Python, Proceedings of the $12^{\text {th }}$ INDIACom; INDIACom-2018; IEEE Conference ID: $4283520185^{\text {th }}$ International Conference on "Computing for Sustainable Global Development", 2018.

16. P. Viola and M. Jones, "Rapid Object Detection using a Boosted Cascade of Simple Features, Conference on Computer Vision and Pattern Recognition", DOI:10.1109/CVPR.2001.990517, 2001.

17. Madhukeerthana, Y. Bevish Jinila, "A review on rough set theory in medical images", Research Journal of Pharmaceutical, Biological and Chemical Sciences, Vol.7, Issue 1, pp.815-822, ISSN : 0975- 8585, 2016.

18. Niveditha.P.R, R.Subhashini, Divya.G: "Recognition And Evaluation Of Facial Expression And Emotion Of Students Using Surveillance Cameras With Thermal Detectors", Organized by IRAJ Research Forum International Conference, ISBN:-978-93-82702-64-1, Hydrebad, March 2014.

19. Dr.R.Subhashini and Niveditha.P.R, "ANALYZING AND DETECTING EMPLOYEE'S EMOTION FOR AMELIORATION OF ORGANIZATIONS", International Conference on Intelligent Computing, Communication \& Convergence(ICCC-2014), Bhubaneswar, Odisha on 27th28th December, 2014. 